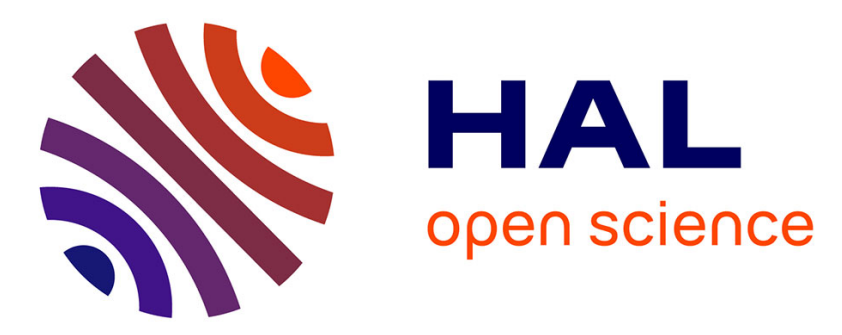

\title{
Sur une analogie de comportement du chrome avec le cuivre dans des amas de quelques atomes formés en emission ionique secondaire
}

\author{
M. Leleyter
}

\section{- To cite this version:}

M. Leleyter. Sur une analogie de comportement du chrome avec le cuivre dans des amas de quelques atomes formés en emission ionique secondaire. Journal de Physique Lettres, 1982, 43 (9), pp.305-313. 10.1051/jphyslet:01982004309030500 . jpa-00232051

HAL Id: jpa-00232051

https://hal.science/jpa-00232051

Submitted on 1 Jan 1982

HAL is a multi-disciplinary open access archive for the deposit and dissemination of scientific research documents, whether they are published or not. The documents may come from teaching and research institutions in France or abroad, or from public or private research centers.
L'archive ouverte pluridisciplinaire HAL, est destinée au dépôt et à la diffusion de documents scientifiques de niveau recherche, publiés ou non, émanant des établissements d'enseignement et de recherche français ou étrangers, des laboratoires publics ou privés. 
Classification

Physics Abstracts

$31.20 \mathrm{P}-36.40-79.20 \mathrm{~N}$

\title{
Sur une analogie de comportement du chrome avec le cuivre dans des amas de quelques atomes formés en émission ionique secondaire
}

\author{
M. Leleyter (*) \\ Groupe de Physique Théorique, Faculté des Sciences, 33, rue Saint-Leu, 80039 Amiens Cedex, France
}

(Reçu le 14 janvier 1982, accepté le 8 mars 1982)

\begin{abstract}
Résumé. - Les intensités des ions secondaires $\mathrm{Cr}_{n}^{+}$émis par un échantillon de chrome sous bombardement ionique primaire présentent les mêmes alternances que celles des ions $\mathrm{Cu}_{n}^{+}$émis par le cuivre : renforcements très marqués des intensités des ions de $n$ impair $(n \leqslant 5)$.

Nous étudions alors l'émission ionique secondaire d'un alliage $\mathrm{CuCr}$, puis nous comparons les intensités des ions émis par des alliages cuivre-aluminium et par le composé $\mathrm{Cr}_{5} \mathrm{Al}_{\mathbf{8}}$ : les renversements d'alternances observés pour les ions $\mathrm{CrCu}_{n}^{+}, \mathrm{Al}_{p} \mathrm{Cu}_{n}^{+}$et $\mathrm{Al}_{p} \mathrm{Cr}_{n}^{+}$par rapport aux ions $\mathrm{Cu}_{n}^{+}, \mathrm{Cr}_{n}^{+}$ et $\mathrm{Al}_{p}^{+}$, permettent de conclure à une analogie de comportement du chrome avec le cuivre, c'est-à-dire à une émission préférentielle des ions positifs homo- ou hétéronucléaires à nombre total d'atomes impair, que ces atomes soient $\mathrm{Cu}, \mathrm{Cr}$ ou $\mathrm{Al}$, donc avec un nombre total pair d'électrons de valence, soit une nouvelle extension de la règle dite de parité à un élément de transition.

Abstract. - The intensities of secondary $\mathrm{Cr}_{n}^{+}$ions emitted from a chromium sample under a primary ionic bombardment, show the same alternations as those of $\mathrm{Cu}_{n}^{+}$ions emitted from copper : very pronounced enhancements of the intensities of $n$-odd ions $(n \leqslant 5)$.

We have studied the secondary ionic emission from $\mathrm{CuCr}$ alloy, and we have compared the intensities of the ions emitted from some copper-aluminium alloys and from the compound $\mathrm{Cr}_{5} \mathrm{Al}_{8}$ : inversions of the alternations for ions $\mathrm{CrCu}_{n}^{+}, \mathrm{Al}_{p} \mathrm{Cu}_{n}^{+}$and $\mathrm{Al}_{p} \mathrm{Cr}_{n}^{+}$in comparison with ions $\mathrm{Cu}_{n}^{+}, \mathrm{Cr}_{n}^{+}$and $\mathrm{Al}_{p}^{+}$, enable us to conclude that an analogy of behaviour exists between chromium and copper, that is to say a preferential emission of the homo- or heteronuclear positive ions with an odd total number of atoms (atoms of $\mathrm{Cu}, \mathrm{Cr}$ or $\mathrm{Al}$ ), therefore with an even total number of valence electrons, namely an additional extension of the "parity rule" to a transition element atom.
\end{abstract}

1. Introduction. - Nous nous proposons dans cet article d'exposer certains résultats expérimentaux permettant de conclure d'une part à une certaine analogie dans les comportements du chrome et du cuivre dans de très petits agrégats et d'autre part à une extension possible de la règle dite de parité à des amas $\mathrm{M}_{p} \mathrm{Cr}_{n}^{+}$(où $\mathrm{M}=\mathrm{Cu}$ ou $\mathrm{Al}$ ) produits en émission ionique secondaire (E.I.S.).

Nous allons examiner tout d'abord l'E.I.S. du cuivre et du chrome purs ou alliés entre eux,

(*) Aussi Laboratoire de Physique des Solides, associé au C.N.R.S. (LA 2), Bâtiment 510, Université Paris-Sud, 91405 Orsay Cedex, France. 
puis les agrégats émis par des alliages du cuivre ou du chrome avec un autre élément, l'aluminium par exemple.

Les expériences ont été menées à l'aide du microanalyseur ionique CAMECA SMI 300 du Laboratoire d'Orsay, qui permet de détecter les agrégats de masses au plus égales à 260 environ et dans lequel les échantillons subissent un bombardement par des ions primaires $\mathrm{Ar}^{+} \mathrm{de} 6,5 \mathrm{keV}$.

2. E.I.S. du cuivre et du chrome. Interprétation. - 2.1 RÉSULTATS EXPÉRIMENTAUX. - Les ions secondaires $\mathrm{Cu}_{n}^{+}$et $\mathrm{Cr}_{n}^{+}$sont représentés figure 1 sur laquelle on a indiqué aussi l'émission d'échantillons d'argent. On peut ainsi se rendre compte de la grande similitude entre les variations des intensités $I\left(\mathrm{Cr}_{n}^{+}\right)$(partie inférieure de la figure) et celles des intensités $I\left(\mathrm{Cu}_{n}^{+}\right)$, c'est-àdire un renforcement très marqué de l'émission des ions à nombre impair d'atomes.

Le comportement en dents de scie des intensités $I\left(\mathrm{Cu}_{n}^{+}\right)$est à présent bien connu $[1,2,3]$ puisque ces alternances ont été mises en évidence jusqu'à $\mathrm{Cu}_{15}^{+}$[3] avec un renforcement toujours très apparent des ions à nombre impair d'atomes, même pour $\mathrm{Cu}_{15}^{+}$. Dans le cas des ions $\mathrm{Cr}_{n}^{+}$, on observe donc un phénomène très voisin. Toutefois, cette similitude semble moins nette pour l'ion $\mathrm{Cr}_{5}^{+}$et peut être un prélude à un changement de comportement.

2.2 INTERPRÉTATION. - Le rapprochement du chrome avec les éléments I-B qui est suggéré par les résultats expérimentaux, peut être justifié par la structure électronique externe du chrome. En effet, ce dernier, à la différence des autres éléments de transition $3 \mathrm{~d}$ auxquels il devrait être normalement apparenté, n'a qu'un seul électron de valence exactement comme le cuivre.

On peut toutefois objecter à ce raisonnement la présence d'une couche $\mathrm{d}$ à moitié remplie dans le chrome alors que dans le cuivre, elle contient 10 électrons. Mais la similitude des deux courbes $I\left(\mathrm{Cu}_{n}^{+}\right)$et $I\left(\mathrm{Cr}_{n}^{+}\right)$montre que la règle dite de parité suivant laquelle les ions secondaires les plus abondants sont ceux qui ont un nombre pair d'électrons de valence [4], règle qui a été

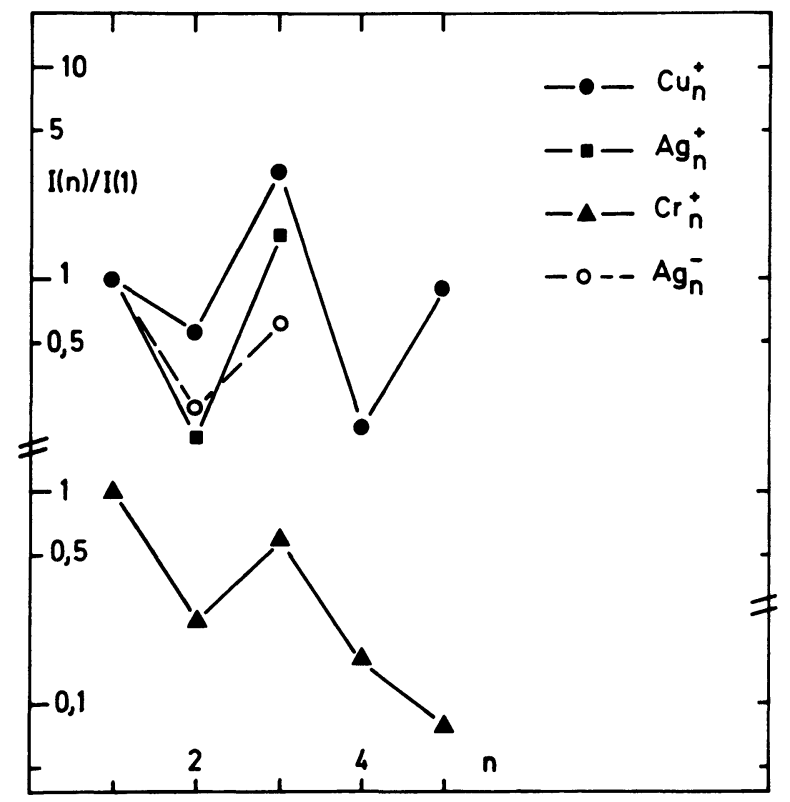

Fig. 1. - E.I.S. des métaux nobles et du chrome : intensités relatives d'émission des ions $\mathrm{X}_{n}^{+}$ou $\mathrm{X}_{n}^{-}$en fonction de $n$. En pointillés, ions $\mathrm{Ag}_{n}^{-}$(mesures de $\mathrm{Krohn}$ [14] avec ions primaires $\mathrm{Cs}^{+}$de $1,1 \mathrm{keV}$ ).

[S.I.M.S. from noble metals and chromium : relative emission intensities of ions $\mathrm{X}_{n}^{+}$or $\mathrm{X}_{n}^{-}$versus $n$. In dashed line, ions $\mathrm{Ag}_{n}^{-}$(measurements of $\mathrm{Krohn}$ [14] with primary $\mathrm{Cs}^{+}$ions of $1.1 \mathrm{keV}$ ).] 
déduite en particulier du comportement des éléments I-B et des alcalins [5] en E.I.S., mais qui a été généralisée à de nombreux autres cas, peut s'appliquer aussi aux ions $\mathrm{Cr}_{n}^{+}$: cela suppose donc que l'on exclut de la catégorie électrons de valence les électrons $3 \mathrm{~d}$ bien que la couche $3 \mathrm{~d}$. $\mathrm{du}$ chrome soit incomplète. On sait toutefois qu'une couche $\mathrm{d}$ à moitié remplie, qui contient donc le nombre maximum d'électrons de spins parallèles, présente une stabilité extrêmement importante [6].

Le cuivre est à présent connu comme ayant, dans les agrégats de quelques atomes, un comportement hydrogénoïde [2, 4], malgré l'existence de ses 10 électrons $3 \mathrm{~d}$. Le résultat de l'expérience (Fig. 1) montre que l'on pourrait tirer la même conclusion quant au chrome quoique la couche $3 \mathrm{~d}$ à moitié remplie de ce dernier soit un peu moins stable que la couche $d$ complète du cuivre. En effet, d'après les données de Slater [7] sur les énergies à un électron des niveaux $3 \mathrm{~d}$ et $4 \mathrm{~s}$, il apparaît que, mis à part le cas du vanadium pour lequel l'écart entre ces 2 niveaux est de 0,19 Rydberg ( 1 Rydberg $=13,6 \mathrm{eV}$ ), le chrome est de tous les éléments $3 \mathrm{~d}$ celui qui a le plus fort écart entre les niveaux $3 \mathrm{~d}$ et $4 \mathrm{~s}$, soit 0,18 Ryd., ce qui le rapproche effectivement du cuivre pour lequel cet écart est de 0,22 Ryd., alors que, par exemple pour le manganèse, l'écart est seulement de 0,07 Ryd. Il semble donc bien légitime quand le nombre d'atomes de chrome est petit, de séparer les électrons $3 \mathrm{~d}$ du chrome des électrons de valence comme dans le cas du cuivre, c'est-à-dire de les inclure dans le cour de l'atome, et par conséquent de considérer que tout se passe comme si le chrome était un élément de type hydrogénoïde. Toutefois, le fait que l'intensité de l'ion $\mathrm{Cr}_{5}^{+}$ soit plus faible que celle de $\mathrm{Cr}_{4}^{+}$, à la différence du cas des ions $\mathrm{Cu}_{n}^{+}$, montre déjà la limite de notre analogie.

2.3 E.I.S. D'UN ALLIAGE $\mathrm{CuCr}$. - On peut essayer de vérifier cette conclusion dans un premier temps en examinant le comportement d'agrégats de cuivre dans lesquels un atome de cuivre est « remplacé » par un atome de chrome, agrégats que l'on obtient par le bombardement ionique d'un alliage $\mathrm{CuCr}$.

Le résultat se trouve sur la figure 2 où sont représentées les intensités des ions secondaires recueillis dans le bombardement d'un alliage à $500 \mathrm{ppm}$ de chrome. Cette très faible teneur, due à la solubilité extrêmement réduite du chrome dans le cuivre, explique que l'on n'ait pu obtenir d'agglomérat contenant plus d'un atome de chrome. En effet, la formation d'un amas est évidemment tributaire de la proximité des éléments dans la cible [8,9]. Un très faible nombre d'atomes de chrome, qui sont donc très dispersés dans la matrice de cuivre, conduit en conséquence à une probabilité extrêmement réduite d'émission en même temps de 2 atomes de chrome.

Le spectre des ions polyatomiques de l'alliage $\mathrm{CuCr}$ nous a fourni deux types d'agrégats : d'une part les ions $\mathrm{Cu}_{n}^{+}$qui ont exactement le mềme comportement que dans le cuivre pur, et d'autre part les ions $\mathrm{CuCr}^{+}$et $\mathrm{Cu}_{2} \mathrm{Cr}^{+}$. Aucun pic n'a pu être détecté pour la masse correspondant à celle de l'ion $\mathrm{Cu}_{3} \mathrm{Cr}^{+}$, alors que l'ion $\mathrm{Cu}_{4}^{+}$, de même nombre d'atomes, est parfaitement visible sur le spectre. L'ion $\mathrm{Cu}_{3} \mathrm{Cr}^{+}$semble donc avoir une très faible probabilité d'émission, et s'il se forme au cours du bombardement, son intensité se situe au-dessous de la limite de sensibilité de notre appareil.

La comparaison des deux courbes de la figure $2: I\left(\mathrm{Cu}_{n}^{+}\right)$(courbe 1) et $I\left(\mathrm{CrCu}_{n}^{+}\right)$(courbe 2) en fonction de $n$, montre un exemple typique de renversement d'alternances puisque, si l'on a bien :

$$
I\left(\mathrm{Cu}_{2}^{+}\right)<\left\{\begin{array}{l}
I\left(\mathrm{Cu}^{+}\right) \\
I\left(\mathrm{Cu}_{3}^{+}\right)
\end{array}\right. \text {et }
$$

on a au contraire :

$$
I\left(\mathrm{CrCu}_{2}^{+}\right)\left\{\begin{array}{l}
>I\left(\mathrm{CrCu}^{+}\right) \\
\gg I\left(\mathrm{CrCu}_{3}^{+}\right) \text {puisque ce dernier n'est même pas détecté } .
\end{array}\right.
$$




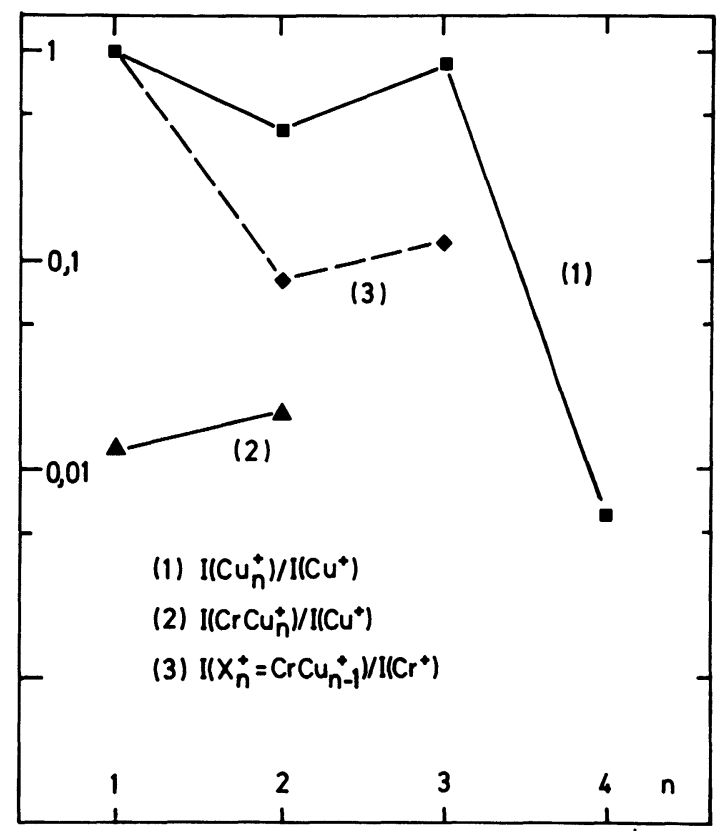

Fig. 2. - Emission secondaire d'un alliage $\mathrm{CuCr}(500 \mathrm{ppm} \mathrm{Cr})$. Les courbes (1) et (2) montrent un exemple typique de renversement d'alternances.

[Secondary emission from an alloy $\mathrm{CuCr}(500 \mathrm{ppm} \mathrm{Cr})$. Curves (1) and (2) show a typical example of inversion of the alternations of $\mathrm{Cu}_{n}^{+}$ions.]

On peut aussi généraliser ce résultat en disant que tout ion positif à nombre total d'atomes impair, que ses atomes soient uniquement de cuivre ou soient de cuivre et de chrome, est émis en plus grande abondance qu'un ion positif à nombre total d'atomes $(\mathrm{Cu}$ ou $\mathrm{Cu}$ et $\mathrm{Cr}$, ou $\mathrm{Cr}$ ) pair. Ceci est résumé sur la figure 2 par la courbe $3: I\left(\mathrm{X}_{n}^{+}\right)$en fonction de $n$ où l'ion $\mathrm{X}_{n}^{+}$représente $\mathrm{CrCu}_{n-1}^{+}$, qui est tout à fait semblable à la courbe 1 des ions homonucléaires $\mathrm{Cu}_{n}^{+}$.

3. E.I.S. d'alliages de cuivre-aluminium ou de chrome-aluminium. - Examinons à présent le cas des alliages de cuivre ou de chrome avec l'aluminium.

3.1 Alliages Cuivre-Aluminium. - L'E.I.S. des alliages $\mathrm{CuAl}$ a déjà été exposée précédemment [10]. Rappelons-en rapidement les principaux résultats.

Les alliages bombardés sont de deux teneurs : $\mathrm{CuAl}$ à $7 \%$ d'aluminium en masse et $\mathrm{AlCu}$ à $4 \%$ de cuivre, en masse également.

3.1.1 Lorsqu'on porte les intensités $I\left(\mathrm{Al}_{p} \mathrm{Cu}_{n}^{+}\right)$en fonction du nombre $n$ d'atomes de cuivre (Fig. 3), on observe successivement 3 renversements d'alternances : en effet, les maxima qui ont lieu pour $n$ impair pour les ions $\mathrm{Cu}_{n}^{+}$et $\mathrm{Al}_{2} \mathrm{Cu}_{n}^{+}$, se produisent au contraire pour $n$ pair dans le cas des ions $\mathrm{AlCu}_{n}^{+}$et $\mathrm{Al}_{3} \mathrm{Cu}_{n}^{+}$. On a donc renversement d'alternances à chaque atome supplémentaire d'aluminium " ajouté » aux amas de cuivre, et ce phénomène se comprend très bien à partir de la règle de parité rappelée plus haut puisque l'aluminium a un nombre impair d'électrons de valence : l'addition d'un nombre impair d'atomes d'aluminium à un ion $\mathrm{Cu}_{n}^{+}$renverse en effet la parité de son nombre total d'électrons. On peut d'ailleurs noter aussi le même phénomène pour les ions négatifs (courbes $\mathrm{Cu}_{n}^{-}$et $\mathrm{AlCu}_{n}^{-}$, Fig. 3). 


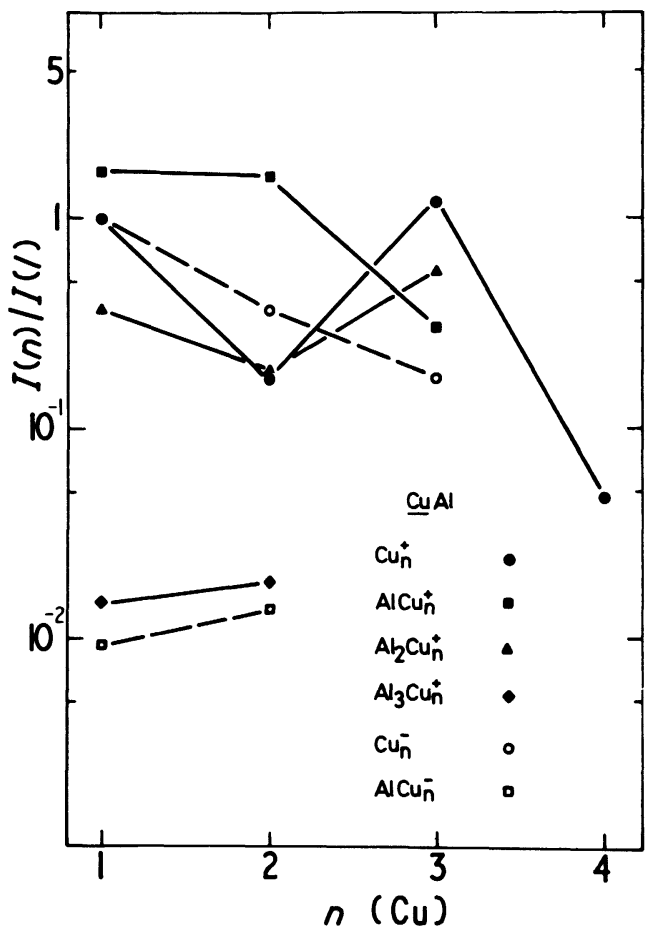

Fig. 3. - Intensités relatives des ions secondaires $\mathrm{Al}_{p} \mathrm{Cu}_{n}^{ \pm}(p=0,1,2,3)$ émis par l'alliage $\mathrm{CuAl}$ en fonction de $n(\mathrm{Cu})$. Les intensités $I\left(\mathrm{AlCu}_{n}^{-}\right)$ont été divisées par 10 pour clarifier la figure.

[Relative intensities of secondary $\mathrm{Al}_{p} \mathrm{Cu}_{n}^{ \pm}(p=0,1,2,3)$ ions emitted from $\mathrm{CuAl}$ alloy versus $n(\mathrm{Cu})$. Intensities $I\left(\mathrm{AlCu}_{n}^{-}\right)$are divided by 10 in order to clarify the figure.]

3.1.2 Lorsqu'on examine les variations de $I\left(\mathrm{Al}_{p} \mathrm{Cu}_{n}^{+}\right)$en fonction du nombre $p$ d'atomes d'aluminium, on observe encore des alternances avec certains renversements, mais le phénomène est moins net que dans le cas précédent (Fig. 4). On peut dire cependant que l'émission des ions $\mathrm{CuAl}_{p}^{+}$et $\mathrm{Cu}_{3} \mathrm{Al}_{p}^{+}$est favorisée quand $p$ est pair $(p=2)$ alors que pour $\mathrm{Cu}_{2} \mathrm{Al}_{p}^{+}$, la courbe devient monotone : le « maximum » en $p=2$ a disparu. L'effet d'alternance est toutefois plus visible pour les ions $\mathrm{CuAl}_{p}^{+}$émis par l'alliage $\mathrm{AlCu}$ du fait de la plus grande concentration d'aluminium dans la matrice qui conduit à une plus grande probabilité d'émission d'amas riches en aluminium.

$\mathrm{Au}$ total, on peut conclure que l'émission des ions $\mathrm{Al}_{p} \mathrm{Cu}_{n}^{+}$est favorisée si le nombre total d'atomes $(n+p)$ est impair et cela même si $n$ ou $p$ sont nuls.

3.2 Alliage Chrome-Aluminium. - 3.2.1 Influence du nombre d'atomes d'aluminium sur les ions $\mathrm{Cr}_{n}^{+}$. - La figure 5 donne les intensités relatives d'émission en fonction de $n(\mathrm{Cr})$, des ions $\mathrm{Cr}_{n}^{+}, \mathrm{AlCr}_{n}^{+}$et $\mathrm{Al}_{2} \mathrm{Cr}_{n}^{+}$, obtenus par le bombardement ionique du composé $\mathrm{Cr}_{5} \mathrm{Al}_{8}$ [11].

On retrouve les alternances typiques du chrome sur les ions $\mathrm{Cr}_{n}^{+}$, quoiqu'un peu gommées par la " dilution » du chrome par l'aluminium. Par ailleurs, on peut dire que dans une certaine mesure, les ions $\mathrm{Al}_{p} \mathrm{Cr}_{n}^{+}$présentent deux renversements successifs des alternances du chrome, bien que de plus en plus estompés. En effet, pour $n=3$, le maximum des ions $\mathrm{Cr}_{n}^{+}$(concavité négative de la courbe) est remplacé pour les $\mathrm{AlCr}_{n}^{+}$par un point anguleux à concavité positive alors que pour $n=2$, la concavité positive de la courbe des ions $\mathrm{Cr}_{n}^{+}$devient négative sur la courbe des ions $\mathrm{AlCr}_{n}^{+}$. On retrouve une concavité positive en $n=2$ sur la courbe des ions $\mathrm{Al}_{2} \mathrm{Cr}_{n}^{+}$. 


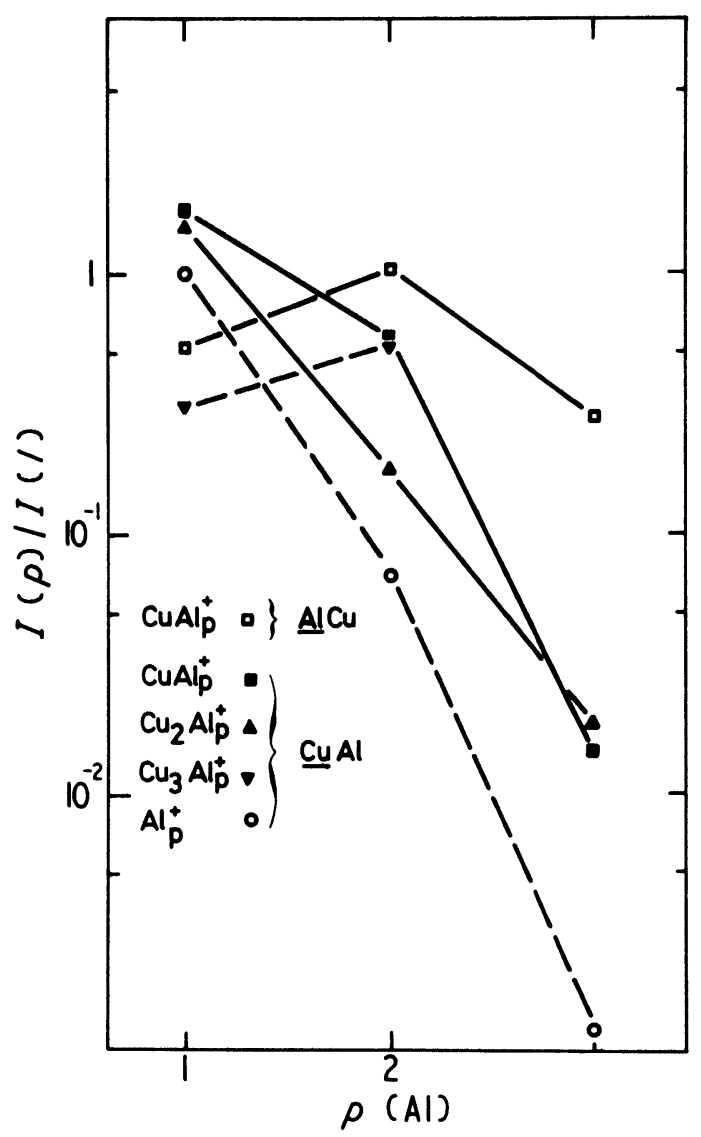

Fig. 4. - Intensités relatives en fonction de $p(\mathrm{Al})$ des ions secondaires $\mathrm{Al}_{p} \mathrm{Cu}_{n}^{+}(n=0,1,2,3)$ émis par les alliages $\mathrm{CuAl}$ et $\mathrm{AlCu}$.

[Relative intensities of secondary $\mathrm{Al}_{p} \mathrm{Cu}_{n}^{+}(n=0,1,2,3)$ ions emitted from $\underline{\mathrm{CuAl}}$ and $\underline{\mathrm{AlCu}}$ alloys, as a function of $p(\mathrm{Al})$.]

Le fait que les alternances des ions $\mathrm{Cr}_{n}^{+}$et leurs renversements soient de moins en moins nets quand on augmente le nombre d'atomes d'aluminium n'est guère surprenant car il est bien évident que les phénomènes ne sont plus aussi simples que dans les amas $\mathrm{CrCu}_{n}^{+}$où, du fait de la présence d'un atome de chrome unique, seuls les électrons $4 s$ étaient susceptibles de jouer un rôle. Ici, au contraire, les électrons $d$ du chrome et les électrons $s$ et $p$ de l'aluminium vont intervenir pour brouiller les alternances.

3.2.2 Ions $\mathrm{CrAl}_{p}^{+}$. - Sur la figure 6, nous avons porté les intensités $I\left(\mathrm{Al}_{p}^{+}\right)$et $I\left(\mathrm{CrAl}_{p}^{+}\right)$en fonction du nombre $p(\mathrm{Al})$ d'atomes d'aluminium. Le fait le plus remarquable est la disparition pour l'ion $\mathrm{CrAl}_{7}^{+}$du maximum visible en $\mathrm{Al}_{7}^{+}$sur la courbe des ions $\mathrm{Al}_{p}^{+}$, du fait de la présence de l'unique atome de chrome dans l'ion. Dans ce cas précis, nous retrouvons bien en effet les conclusions obtenues pour les ions $\mathrm{CuAl}_{p}^{+}$et $\mathrm{CrCu}_{n}^{+}$, c'est-à-dire l'accord avec la règle de parité.

4. Conclusion. - Nous voyons donc que les propriétés expérimentales des ions $\mathrm{Cr}_{n}^{+}$peuvent inciter à classer le chrome dans la même catégorie que le cuivre ou l'argent tant que le nombre $n$ reste petit. Remarquons cependant qu’à la différence du cuivre ou de l'argent, nous n'avons pas 


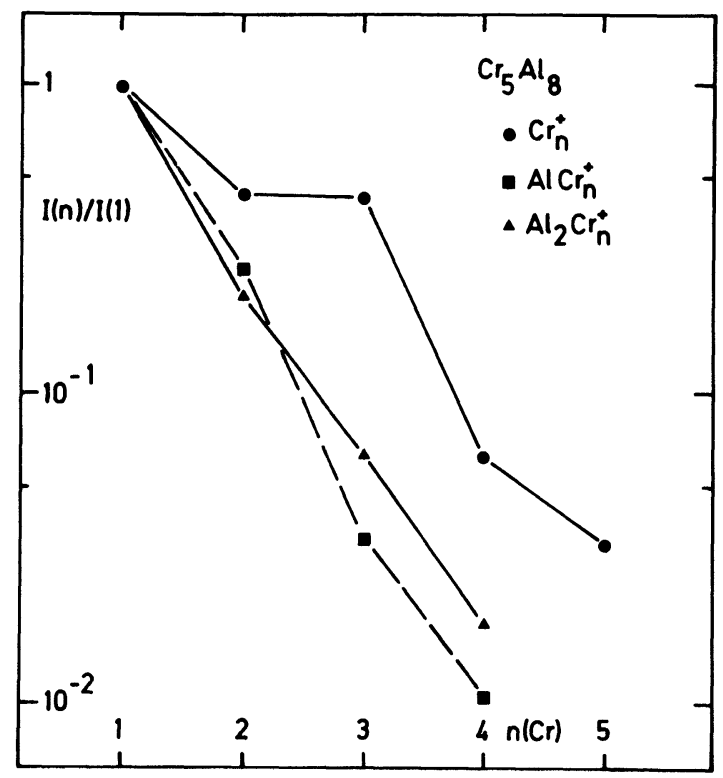

Fig. 5. - Intensités relatives rapportées à $I\left(\mathrm{Cr}^{+}\right)$, en fonction de $n(\mathrm{Cr})$, des ions secondaires $\mathrm{Al}_{p} \mathrm{Cr}_{n}^{+}$émis par l'alliage $\mathrm{Cr}_{5} \mathrm{Al}_{8}$.

[Relative intensities, related to $I\left(\mathrm{Cr}^{+}\right)$, of secondary $\mathrm{Al}_{p} \mathrm{Cr}_{n}^{+}$ions emitted from $\mathrm{Cr}_{5} \mathrm{Al}_{8}$ alloy, as a function of $n(\mathrm{Cr})$.]

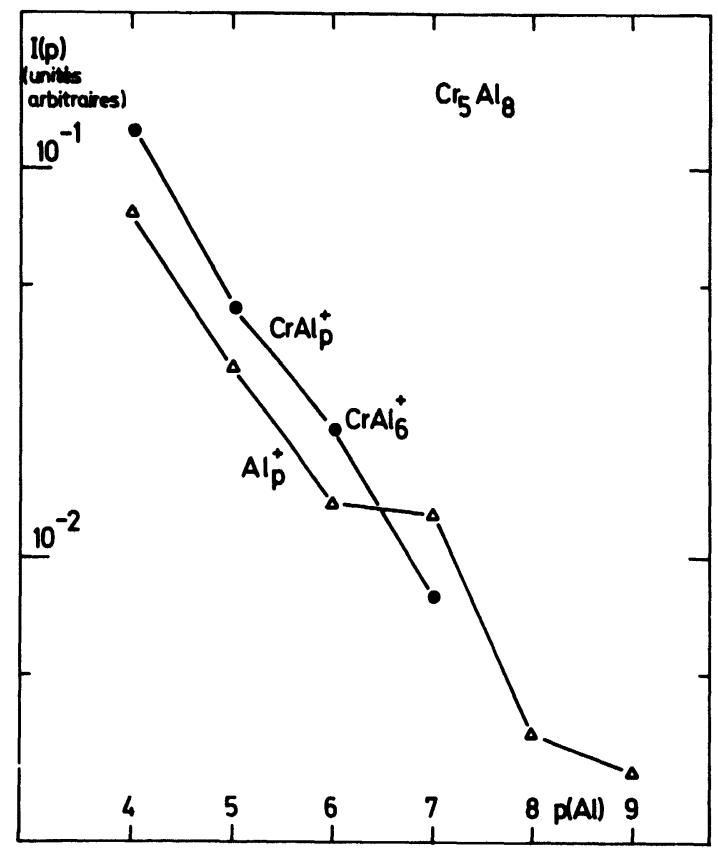

Fig. 6. - Emission des ions secondaires $\mathrm{Al}_{p}^{+}$et $\mathrm{CrAl}_{p}^{+}$en fonction de $p(\mathrm{Al})$ par l'alliage $\mathrm{Cr}_{5} \mathrm{Al}_{8}$. Le « maximum " en $\mathrm{Al}_{7}^{+}$disparait sur la courbe des ions $\mathrm{CrAl}_{p}^{+}$.

[Emission of secondary $\mathrm{Al}_{p}^{+}$and $\mathrm{CrAl}_{p}^{+}$ions from $\mathrm{Cr}_{5} \mathrm{Al}_{8}$ alloy versus $p(\mathrm{Al})$. The "maximum " at $\mathrm{Al}_{7}^{+}$ disappears from the curve of $\mathrm{CrAl}_{p}^{+}$ions.] 
obtenu d'ions $\mathrm{Cr}_{n}^{-}$en E.I.S. à l'exception de l'ion $\mathrm{Cr}^{-}$. L'affinité électronique mesurée du chrome est en effet bien inférieure à celle de l'argent (respectivement $0,85 \mathrm{eV}$ au lieu de 1,30 eV [12]). En outre les mesures de moments magnétiques de Kant et Mc Caffrey [13] donnent pour $\mathrm{Cr}_{2}$ un état fondamental ${ }^{3} \Sigma$ magnétique qui ne permet donc pas de conclure en faveur ou non du caractère hydrogénoïde du chrome alors que les mesures sur $\mathrm{Cu}_{2}, \mathrm{Ag}_{2}$ et $\mathrm{Au}_{2}$ donnent un état ${ }^{1} \Sigma$ non magnétique qui peut s'accorder avec un caractère typiquement hydrogénoïde.

D'un autre côté, l'E.I.S. du composé $\mathrm{Cr}_{5} \mathrm{Al}_{8}$ montre bien qu'il devient aventureux d'assimiler totalement les agrégats de chrome à ceux du cuivre en supposant négligeables les effets de bande $\mathrm{d}$ comme nous l'avons fait dans le cas des ions homonucléaires $\mathrm{Cr}_{n}^{+}$avec $n<5$. Cependant, malgré ces réserves, lorsqu'un seul atome de chrome est engagé dans un ion $\mathrm{Cu}_{n}^{+}$ou $\mathrm{Al}_{p}^{+}$où les effets de bande $\mathrm{d}$ restent imperceptibles, on peut effectivement conclure à l'analogie du chrome et du cuivre, ce qui peut constituer une approximation commode pour interpréter certaines expériences.

Enfin, on a pu aussi constater que la règle de parité est vérifiée qualitativement pour les amas homo- ou hétéronucléaires de chrome à condition de considérer que le chrome possède un seul électron de valence $4 \mathrm{~s}$ comme le cuivre, ses 5 électrons d étant inclus dans le cour de l'atome. (Nous indiquons en appendice un résultat voisin mais dans le cas du nickel.) On peut dès lors appliquer aux agrégats $\mathrm{Cr}_{n}$ le même type de calcul dans l'approximation de Hückel qui a déjà été effectué pour les amas $\mathrm{Cu}_{n}[2,4]$ pour étudier les stabilités relatives en fonction de $n$ et vérifier quantitativement cette fois la règle de parité. Toutefois, il est bien évident qu'une étude de ce problème ne saurait être complète et satisfaisante que si les calculs prennent en compte à la fois les électrons d et $\mathrm{s}$ du chrome ou du cuivre.

Appendice. - Nous pouvons mentionner ici un phénomène très semblable à celui que nous avons exposé dans cet article et qui a été mis en évidence par Rodriguez-Murcia et Beske dans leur belle série d'expériences sur divers alliages $\mathrm{CuNi}$ [3]. C'est ainsi que les intensités des ions $\mathrm{Ni}_{p} \mathrm{Cu}_{n}^{+}$ émis en particulier par 2 alliages $\mathrm{CuNi}$, l'un à $5 \%$ atomique de $\mathrm{Ni}$ et l'autre à $10 \%$ at., montrent très nettement deux renversements successifs d'alternances des ions $\mathrm{Cu}_{n}^{+}$lorsque l'on " ajoute » un puis deux atomes de nickel aux ions $\mathrm{Cu}_{n}^{+}$, de sorte que les alternances des ions $\mathrm{Ni}_{2} \mathrm{Cu}_{n}^{+}$sont les mêmes (pour $n \leqslant 7$ ) que celles des $\mathrm{Cu}_{n}^{+}$(ions de $n$ impair renforcés) alors que celles des ions $\mathrm{NiCu}_{n}^{+}$(pour $n \leqslant 8$ ) correspondent au renforcement des ions de $n$ pair. Cela est d'autant plus surprenant que les intensités des ions homonucléaires $\mathrm{Ni}_{p}^{+}$relevées dans les mêmes expériences décroissent de manière parfaitement monotone (pour $p \leqslant 10$ ).

Ce résultat expérimental relatif aux ions hétéronucléaires $\mathrm{Ni}_{p} \mathrm{Cu}_{n}^{+}$pourrait en fait trouver une interprétation simple dans la ligne de ce que nous indiquions plus haut ; en effet, si l'on se réfère à des calculs ab-initio récents sur la structure du dimère $\mathrm{Ni}_{2}$ [15], ceux-ci montrent que les états de $\mathrm{Ni}_{2}$ situés les plus bas en énergie proviennent en réalité de la combinaison de deux atomes de nickel dans la configuration $3 d^{9} 4 s^{1}$ et non dans la configuration $3 d^{8} 4 s^{2}$ comme on aurait pu le supposer, et les auteurs en concluent que le schéma de base des états les plus stables de $\mathrm{Ni}_{2}$ est celui d'une liaison par les électrons $4 \mathrm{~s}$ analogue à celle des métaux alcalins ou de $\mathrm{H}_{2}$.

Dans ces conditions, tout se passerait donc comme si l'atome de nickel n'apportait à l'amas $\mathrm{Cu}_{n}^{+}$ qu'un seul électron 4s, les 9 électrons d restant liés au cour de l'atome de sorte que la règle de parité se trouverait encore vérifiée dans ce cas (mais non pas dans celui des ions homonucléaires $\mathrm{Ni}_{p}^{+}$qui semble ressortir d'une autre interprétation). Là encore, seule une étude théorique complète serait à même d'éclaircir la question.

On peut finalement conclure que tout ce qui précède met en évidence la très grande différence qui peut exister dans le comportement d'un élément de transition suivant qu'il est engagé dans un très petit amas ou qu'il constitue un solide massif. . 


\section{Bibliographie}

[1] Woodyard, J. R., XV Ann. Conf. Mass Spectrometry (1967) Denver, p. 264, cité par Perovic, B., The Internat. School on Mass Spectrom. (1969), Ljubljana, Yougoslavie.

[2] JoYes, P., J. Phys. Chem. Solids 32 (1971) 1269-75.

[3] RodrigueZ-Murcia, H., Beske, H. E., Dissertation JUL 1292 (1976), Kernforschungsanlage, Jülich, Allemagne.

[4] Leleyter, M., Thèse d'Etat, Orsay (1975).

[5] Leleyter, M., Joyes, P., Radiat. Eff. 18 (1973) 105-110.

[6] Gray, H. B., Electrons and Chemical Bonding (W. A. Benjamin, New York) 1964, p. 35.

[7] Slater, J. C., Phys. Rev. 98, no 4 (1955) 1039-45.

[8] Laurent, R., Proc. Intern. Conf. Ion Sputtering and Related Phenomena (1972), Garching (Munich, Allemagne) 153-6; publié dans Radiat. Eff. (1973).

[9] BernheIm, M., Radiat. Eff. 18 (1973) 231-4.

[10] Leleyter, M., Joyes, P., J. Phys. B 7, no 4 (1974) 516-28.

[11] $\mathrm{Cr}_{5} \mathrm{Al}_{8}$ : composé préparé par M. Pichoir, ONERA, Châtillon/s/Bagneux, France.

[12] Hotop, H., Bennett, R. A., Lineberger, W. C., J. Chem. Phys. 58, no 6 (1973) 2373.

[13] Kant, A., MCCAfFrey, F., Proceed. Internat. Conf. Mass Spectrometry, 31 août-4 sept. 1970, Bruxelles, Belgique.

[14] Krohn, V. E., J. Appl. Phys. 33, no 2 (1962) 3523-5.

[15] Wood, C., Doran, M., Hillier, I. H., Guest, M. F., Faraday Symposium no 14 (1980) 159-169. 